\title{
Investigar las representaciones de la didáctica de las ciencias sociales en la formación de maestros/as: El caso de la educación infantil ${ }^{1}$
}

Recepción: 20/03/2020 | Revisión: 18/05/2020 | Aceptación: 29/05/2020 | Preprint: 07/12/2020 | Publicación: 01/10/2021

\author{
Diego GARCÍA MONTEAGUDO \\ Universitat de València \\ Diego.Garcia-Monteagudo@uv.es \\ https://orcid.org/0000-0003-0505-0608
}

\begin{abstract}
Resumen: El objetivo del presente trabajo es analizar las representaciones que 134 estudiantes del Grado de Maestro/a de Educación Infantil tienen acerca de la Didáctica de las Ciencias Sociales con la finalidad de conocer los elementos que intervendrán en sus prácticas docentes en el futuro. Para ello, el trabajo se divide en dos partes. En la primera, se expone el marco teórico compuesto de dos subapartados. Por un lado, se sintetizan los rasgos más relevantes de la Didáctica de las Ciencias Sociales y su enseñanza, focalizándose en la Educación Infantil. Por otro lado, se argumenta sobre la teoría de las representaciones sociales como enfoque teórico y metodológico para desarrollar una praxis didáctica que fomente la investigación y la reflexión del futuro profesorado. En la segunda parte, se da cuenta del método de trabajo y de los principales resultados, que evidencian un cambio de representación sobre las Ciencias Sociales y su utilidad posterior en la docencia en Educación Infantil, que se plasma en las temáticas de sus propuestas didácticas. Finalmente, las conclusiones recopilan las ideas más relevantes y algunas propuestas para investigar en el futuro acerca de la formación docente.
\end{abstract}

Palabras clave: representaciones; didáctica de las ciencias sociales, educación infantil; formación docente.

\section{REPRESENTATIONS OF SOCIAL SCIENCES DIDACTICS IN TEACHER TRAINING: THE CASE OF EARLY YEARS EDUCATION}

Abstract: The aim of this paper is to analyze the representations that 134 students of the Early Years Education Degree have about the Didactics of Social Sciences in order to know the elements that will intervene in their teaching practices in the future. To this end, the work is divided into two parts. In the first part, the theoretical framework is presented, consisting of two subsections. On the one hand, the most relevant features of Social Science Didactics and its teaching are synthesized, focusing on Early Years Education. On the other hand, the theory of social representations is discussed as a theoretical and methodological approach to develop a didactic praxis that promotes research and reflection of future teachers. In the second part, we present the working method and the main results, which show a change in the representation of the Social Sciences and their subsequent usefulness in early years education, reflected in the themes of their didactic proposals. Finally, the conclusions gather the most relevant ideas and some proposals for future research on teacher training.

Keywords: representations; teaching of social sciences; childhood education; teacher training.

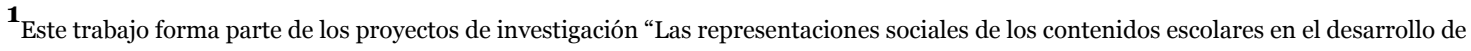
lascompetencias docentes” (PGC2018-094491-B-C32), financiado por el Ministerio de Ciencia, Innovación y Universidades y cofinanciado con fondos FEDER de la UE, y del proyecto "Educación y formación ciudadana del profesorado iberoamericano: conocer la representación del saber geográfico e histórico para promover una praxis escolar crítica” (GV/2021/o68), financiado por la Consellería d’ Innovació, Universitats, Ciència i Societat Digital de la Generalitat Valenciana
} 


\section{INVESTIGAR LES REPRESENTACIONS DE LA DIDÀCTICA DE LES CIÈNCIES SOCIALS EN LA FORMACIÓ DE MESTRES: EL CAS DE L'EDUCACIÓ INFANTIL}

Resum: L'objectiu d'aquest estudi és analitzar les representacions que tenen 134 estudiants del Grau de Mestre d'Educació Infantil sobre la Didàctica de les Ciències Socials per conèxer els elements que intervindran en les seves futures pràctiques docents. Aquest treball es divideix en dues parts. A la primera, s'exposa el marc teòric, dividit en dos subapartats. D'una banda, se sintetitzen els trets més rellevants de la Didàctica de les Ciències Socials i el seu ensenyament, centrant-se en l'Educació Infantil. De l'altra, s'argumenta sobre la teoria de les representacions socials com enfocament teòric i metodològic per desenvolupar una praxi didàctica que fomenti la investigació i la reflexió del futur professorat. A la segona part, s'explica el mètode de treball i els resultats principals, que evidencien un canvi de representació sobre les Ciències Socials i la seva utilitat posterior a la docència en Educació Infantil, que es plasma en les temàtiques de les seves propostes didàctiques. Finalment, les conclusions recopilen les idees més rellevants i algunes propostes per investigar en el futur sobre la formació docent.

Paraules clau: representacions; didàctica de les ciències socials, educació infantil; formació docent.

\section{Introducción}

El objetivo de este estudio es analizar y reflexionar sobre la concepción que el futuro profesorado tiene acerca de la Didáctica de las Ciencias Sociales en el Grado de Maestro/a de Educación Infantil de una universidad española. La finalidad es conocer las ideas previas que tiene el alumnado en formación sobre esta disciplina para promover una enseñanza ajustada y crítica con la representación que tienen acerca de las ciencias sociales y su enseñanza en la etapa de Educación Infantil. En España es la primera etapa del sistema educativo español, tiene carácter voluntario y comprende a niños y niñas de 0 a 6 años de edad. La enseñanza se regula por la Ley Orgánica de Educación (LOE) de 2006 y se organiza en dos niveles: el primero de 0 a 3 años, que se cursa en escuelas infantiles y el segundo de 3 a 6 años, que se cursa en colegios y tiene carácter gratuito. En esta investigación nos interesa conocer la representación en Ciencias Sociales de los futuros maestros y maestras que impartirán docencia en el segundo ciclo de Educación Infantil ( 3 a 6 años), ya que la formación para ejercer en escuelas de Infantil se realiza mediante un curso de Técnico en Educación Infantil, en centros no universitarios.

El cuestionario inicial que respondieron los/las 134 estudiantes de la asignatura Didáctica de las Ciencias Sociales en la Educación Infantil ha aportado los primeros datos para orientar la estrategia docente de esa asignatura, que se imparte en el cuarto curso del Grado de Maestro/a en Educación Infantil. Aunque no es la primera vez que el alumnado tiene contacto con las Ciencias Sociales en sus estudios universitarios, se pretende conocer su concepción de las Ciencias Sociales (Geografía, Historia e Historia del arte), si bien no de manera explícita para evitar el condicionamiento de los resultados. Así se puede entrever si sus respuestas son parte de una representación más amplia sobre estas disciplinas y cómo orientan su práctica docente en los niveles de Educación Infantil al inicio de la asignatura. En el segundo ciclo de Educación Infantil la competencia para regular los objetivos, competencias, contenidos y criterios de evaluación del currículo recae sobre el Ministerio de Educación (Real Decreto 1630/2006, de 29 de diciembre, por el que se establecen las enseñanzas mínimas del segundo ciclo de Educación Infantil), si bien es cierto que las comunidades autónomas completan esa regulación mediante decretos específicos. En el caso de la Comunidad 
Valenciana, región en la que se ha desarrollado esta investigación, el Decreto 38/2008, del Consell, por el que se establece el currículo del segundo ciclo de la Educación Infantil en la Comunitat Valenciana, concreta el decreto nacional antes mencionado.

Con la pretensión de explicar estos resultados de manera razonada, se ha dividido el trabajo en dos partes bien diferenciadas. En la primera se exponen los fundamentos teóricos de la investigación con unas referencias precisas a la Didáctica de las Ciencias Sociales en la Educación Infantil y la argumentación de la teoría de las representaciones sociales como marco de su enseñanza en la formación docente (Castorina, 2017; Lobato, 2013; Moscovici y Hewstone, 1986). En la segunda parte, se presenta la metodología de la investigación y la interpretación de los resultados previos y posteriores de la representación que tiene el alumnado en formación sobre la Didáctica de las Ciencias Sociales durante el transcurso de la asignatura. En las conclusiones se recogen las ideas más relevantes y algunas anotaciones para continuar con esta línea de investigación en próximos trabajos.

\section{La construcción de una Didáctica de las Ciencias Sociales desde sus representaciones en la formación de maestros/as de Educación Infantil}

Al abordar la enseñanza de las Ciencias Sociales y su didáctica en la formación inicial de maestros/ as de Educación Infantil se deben conocer las investigaciones previas que han analizado el estado de la cuestión de esta área de conocimiento, así como algunas publicaciones científicas que han trabajado en una línea similar. Además, se debe indicar el posicionamiento teórico y metodológico que se defiende en este trabajo, que como estudio intensivo de casos se enmarca en la teoría de las representaciones sociales y su relación con la Didáctica de las Ciencias Sociales para promover una enseñanza crítica en la formación del profesorado. Eso es lo que se va a presentar en los dos subapartados siguientes.

\subsection{Didáctica de las Ciencias Sociales y su enseñanza en la Educación Infantil}

A escala internacional no son abundantes los estudios sobre la concepción de la Didáctica de las Ciencias Sociales en el ámbito de la Educación Infantil por parte del profesorado (Hernández, 2013, 2014; Hernández y Pagès, 2014; Morales y Santana, 2019), si bien es cierto que es una línea de investigación con bastante desarrollo en Educación Primaria y Secundaria (Gómez y Núñez, 2006; Pagès, 1996; Santisteban, 2005; Sebastià, 2014). En el caso de la Educación Infantil, se dispone de manuales de referencia (Aranda, 2016; Feliú y Jiménez, 2015; Rivero, 2011; Rodríguez, 1995; Tonda, 2001) que han sintetizado las orientaciones principales sobre la enseñanza de las Ciencias Sociales (Geografía e Historia) en esta etapa y constituyen una referencia fundamental para la formación universitaria.

A la situación anterior se añade la escasa preocupación de las investigaciones en Ciencias Sociales por la infancia, que ha dejado paso a una visión cognitiva de la socialización en los currículos escolares (Aranda, 2016; Bugental y Goodnow, 1998; Cuenca, 2008) y ha sido duramente criticada por considerar a niños y niñas como sujetos pasivos (Corsaro, 1997; James et al., 1998; Simkin y Becerra, 2013). En la Educación Infantil, las Ciencias Sociales nucleares (Geografía e Historia) se han identificado con el conocimiento del entorno desde una perspectiva psicoevolutiva que 
se desprende de un código pedagógico (Mateos, 2001, 2008), lo que choca con las posibilidades de desarrollar el pensamiento geográfico e histórico en Educación Infantil y sucesivas etapas (Calvani, 1988; Canet, Morales y García-Monteagudo, 2018; Catling, 1993; Cooper, 2002; Donet, 2016; Martin y Owens, 2005; Massey, 2005; Palmer y Birch, 2004; Rivero y Gil, 2011; Trepat, 2011). Además, la enseñanza de la Geografía y de la Historia en la etapa de Educación Infantil ofrecen posibilidades didácticas como la contribución al desarrollo de la personalidad del niño a través de su integración con el medio en el que vive (Tonda, 2009). Esto hace posible la construcción escolar de una cosmovisión del mundo si las estrategias didácticas se realizan desde el constructivismo social (Wood y Holden, 2007). En las definiciones siguientes se aprecia que las Ciencias Sociales permiten comprender la actividad de las personas y su relación con el entorno, un hecho que tiene una aplicación directa a la Educación Infantil, pues el marco curricular para el segundo ciclo de Educación Infantil se ha centrado en presentar contenidos vinculados a la vida de los niños y niñas en su medio desde edades tempranas (Aranda, 2016).

Ciencias Sociales son todas las que estudian las actividades del ser humano en sociedad tanto en el pasado como en el presente, y las relaciones e interacciones con el medio y el territorio donde se han desarrollado o desarrollan en la actualidad (Benejam, 1993, p. 342, en Tonda, 2001).

Las ciencias sociales deben plantearse como experiencias científicas de humanidad, útiles para afrontar retos del presente. Los saberes sociales, históricos y geográficos son tanto más útiles y funcionales que cualquier otro, en tanto en cuanto inciden en la formación de una ciudadanía con criterio, el activo más importante al que debe aspirar cualquier comunidad (Hernández, 2008, p. 8).

De las dos definiciones anteriores se pueden extraer algunas ideas relevantes para la formación en Ciencias Sociales de maestros y maestras de Educación Infantil. En la primera acepción, se enfatiza la variedad de disciplinas que componen el campo de las Ciencias Sociales y que comparten un objeto de estudio similar, así como una serie de metodologías afines. Esto es importante porque el alumnado universitario en formación ya ha cursado otras materias de este campo social (Psicología, Sociología, Pedagogía...) que le han proporcionado una serie de conocimientos sobre el ser humano y la educación. Sin embargo, esa pluralidad disciplinar no se corresponde con un marco conceptual único, ya que cada ciencia incorpora una terminología específica en función de su objeto de estudio más característico. Para el caso de las Ciencias Sociales (Geografía e Historia) en la Educación Infantil, los contenidos se enfocan hacia el estudio del pensamiento social (Aranda, 2016), un aspecto que conecta bien con la definición de Hernández (2008), en la que el autor destaca la funcionalidad de los aprendizajes geográficos e históricos y la formación ciudadana del alumnado.

En consecuencia, la adquisición del pensamiento social en el segundo ciclo de Educación Infantil se consigue mediante la planificación y el desarrollo de propuestas didácticas con carácter global y la complementariedad entre las tres áreas que componen el currículo. La primera se denomina "Conocimiento de sí mismo y autonomía personal" y comprende la construcción de la identidad y el desarrollo de relaciones afectivas. La segunda es el "Conocimiento del entorno" que hace referencia a los contextos en los que se desarrolla la vida y las actividades de los niños y niñas. Y la tercera, "Lenguajes: comunicación y representación", establece el desarrollo de distintos lenguajes 
para posibilitar la consolidación de las relaciones sociales, la comprensión de la realidad y la interacción con los distintos contextos. De estas tres áreas hemos seleccionado los objetivos que más se corresponden con las Ciencias Sociales (Geografía e Historia), que coinciden genéricamente con el área de Conocimiento del Entorno (Tabla 1):

\begin{tabular}{|l|}
\hline \multicolumn{1}{|c|}{ Objetivos curriculares más relacionados con las Ciencias Sociales } \\
\hline $\begin{array}{l}\text {-Observar y explorar de forma activa su entorno, generando interpretaciones sobre algunas situaciones y } \\
\text { hechos significativos y mostrando interés por su conocimiento. }\end{array}$ \\
\hline $\begin{array}{l}\text {-Relacionarse con los demás, de forma cada vez más equilibrada y satisfactoria, interiorizando } \\
\text { progresivamente las pautas de comportamiento social y ajustando su conducta a ellas. }\end{array}$ \\
\hline $\begin{array}{l}\text {-Conocer distintos grupos sociales cercanos a su experiencia, algunas de sus características, } \\
\text { producciones culturales, valores y formas de vida, generando actitudes de confianza, respeto y aprecio. }\end{array}$ \\
\hline $\begin{array}{l}\text {-Conocer y valorar los componentes básicos del medio natural y algunas de sus relaciones, cambios y } \\
\text { transformaciones, desarrollando actitudes de cuidado, respeto y responsabilidad en su conservación. }\end{array}$ \\
\hline
\end{tabular}

Tabla 1. Objetivos más afines a las Ciencias Sociales extraídos del RD 1630/2006 que regula las enseñanzas mínimas del segundo ciclo de Educación Infantil en España.

Con respecto a los contenidos más propios de las Ciencias Sociales en el segundo ciclo de Educación Infantil, se pueden encontrar en el tercer bloque dedicado a la cultura y la vida social. En la siguiente tabla (Tabla 2) se han seleccionado los seis más afines al pensamiento social:

\begin{tabular}{|l|}
\hline Bloque 3. Cultura y vida en sociedad \\
\hline -La familia y la escuela como primeros grupos sociales de pertenencia. Toma de conciencia de la \\
necesidad de su existencia y funcionamiento mediante ejemplos del papel que desempeñan en la vida \\
cotidiana. Valoración de las relaciones afectivas que en ellos se establecen. \\
\hline -Observación de necesidades, ocupaciones y servicios en la vida de la comunidad. \\
\hline -Incorporación progresiva de pautas adecuadas de comportamiento, disposición para compartir y \\
resolver conflictos cotidianos mediante el diálogo de forma progresivamente autónoma, atendiendo \\
especialmente a la relación equilibrada entre niños y niñas. \\
\hline -Reconocimiento de algunas señas de identidad cultural del entorno e interés por participar en \\
actividades sociales y culturales. \\
\hline -Identificación de algunos cambios en el modo de vida y las costumbres en relación con el paso del \\
tiempo. \\
\hline $\begin{array}{l}\text {-Interés y disposición favorable para entablar relaciones respetuosas, afectivas y recíprocas con niños y } \\
\text { niñas de otras culturas. }\end{array}$ \\
\hline
\end{tabular}

Tabla 2. Contenidos propios de las Ciencias Sociales del área de Conocimiento del entorno extraídos del RD 1630/2006 que regula las enseñanzas mínimas del segundo ciclo de Educación Infantil en España. 
Como se deduce de los objetivos y contenidos presentados anteriormente, el currículo está impregnado de un enfoque socializador que se concreta en la educación en valores. Mediante este enfoque se pretende generar o cambiar actitudes desde la infancia mediante el refuerzo social, esto es, una modalidad de motivación extrínseca que intenta crear actitudes con el desarrollo de rutinas y hábitos diarios tanto en la escuela como en la familia (Ortega, 1986). Se debe tener en cuenta, entre otros aspectos, que la consideración de neutralidad que se hace en dicho marco curricular por los medios de comunicación (Parra y Segarra, 2011) puede obstaculizar la construcción social de las identidades y la relación de niños y niñas con su entorno. De esta manera se hace necesario abordar una enseñanza crítica que valore las ideas previas del futuro profesorado desde las representaciones sociales y su relación con la didáctica en este ámbito de conocimiento, como se verá en el siguiente apartado.

\subsection{Las representaciones sociales como teoría y método para la praxis escolar}

El marco teórico y metodológico de las representaciones sociales permite acceder a sus dimensiones simbólicas, culturales y prácticas (Jodelet, 2009) de los fenómenos sociales que son objeto de estudio de la Didáctica de las Ciencias Sociales (Benejam, 1993; Casiello, 2012; Hernández, 2008; Souto, 1999) que para el caso de la Educación Infantil la hemos adaptado del manual universitario de Ana María Aranda (2016), tal y como se comprueba a continuación:

En esta área de conocimiento el objetivo fundamental es desarrollar el pensamiento social de niños/as, capacitarles para el análisis de la realidad para que pueda y quiera participar en ella, por medio de propuestas didácticas concebidas y desarrolladas desde la globalidad de la acción y de los aprendizajes, así como desde la complementariedad de las áreas. (Adaptación de Aranda, 2016, pp. 41-46)

En la definición anterior no se prescinde de la educación en valores que consta en el marco curricular, pues los valores son una cualidad del ser humano que guían su comportamiento en la vida desde la infancia (Raths, 1976). Como los valores surgen de la experiencia y pueden ser modificados con el paso del tiempo y la adquisición de vivencias y experiencias que se circunscriben a un contexto social y cultural global, el enfoque de las representaciones sociales y la subjetividad emanada de posicionamientos fenomenológicos (Pillet, 2004) resulta idóneo para conocer la actitud del futuro profesorado ante situaciones específicas docentes en la etapa de Infantil. De hecho, es importante reflexionar sobre la representación del saber (en este caso las Ciencias Sociales) porque es uno de los tres componentes de la enseñanza del futuro profesorado, junto con el dominio afectivo-emocional y práctico (Pontes et al., 2012).

En consecuencia, las representaciones sociales ayudan a conocer el significado de las fuentes y de los contextos de información que inciden en la visión que tiene el alumnado universitario sobre las Ciencias Sociales y su didáctica al inicio del curso. En el currículo del segundo ciclo de Educación Infantil la concepción espacial se reduce al espacio geométrico (Rivero y Gil, 2011) y la utilidad de la historia se identifica con el conocimiento de los errores del pasado y el aprendizaje de lo que no se debe hacer (Barton y Levstik, 2004). Si no se cuestionan estas determinadas formas de entender las Ciencias Sociales (Geografía e Historia) se perpetuará un modelo de currículo técnico (Apple, 2000; Canella y Radhika, 2005; Giroux, 1988) que defenderá una cosmovisión conservadora de la sociedad, bajo la apariencia de cierta neutralidad (Pagès, 1994). El modelo técnico puede 
ser el más extendido en la práctica del futuro profesorado de Educación Infantil, ya que una de las mayores preocupaciones de este profesorado en formación era mostrar los contenidos de forma interesante y con una cierta vinculación con la vida de los niños (Barton et al., 2004). Por ello es relevante que la identidad docente se construya en sintonía a la epistemología y la sociología de la ciencia para que conozcan modelos didácticos innovadores (Porlán et al., 1998), en los que sean conscientes de sus concepciones, actitudes y prácticas que se derivan de una determinada representación de la Didáctica de las Ciencias Sociales.

A partir de la relación entre la ciencia y el conocimiento de sentido común (Moscovici, 2007) las representaciones sociales son un marco de referencia para introducir la subjetividad de la enseñanza de la Geografía y de la Historia en la asignatura de Didáctica de las Ciencias Sociales. En el caso de la Geografía, se ha unido a la Psicología a partir del giro del comportamiento (Capel, 1973) mediante estudios de la Geografía de la Percepción y del Comportamiento, que posteriormente han mostrado que la actividad que las personas proyectan en el espacio geográfico es resultado de una representación sobre un determinado contexto en el que se crea el estímulo que genera dicha representación (Sammut et al., 2015). En el caso de la Historia, el giro cultural ha refrendado la dimensión simbólica y representacional de los procesos históricos (Chartier, 1996), otorgándose una mayor importancia a algunos agentes históricos que tradicionalmente han contado con menor representación en el modelo de historias generales (Maestro, 2002). Con estos presupuestos epistemológicos que chocan con el enfoque psicoevolutivo presente en el currículo de Educación Infantil se apuesta por conocer la representación social de la Didáctica de las Ciencias Sociales entre el profesorado en formación de esta etapa educativa.

\section{Diseño de la experiencia}

Todas las actividades que forman parte del proyecto docente y son necesarias para impartir la asignatura obligatoria a la que nos hemos referido en apartados anteriores, cumplen con las directrices generales que se establecen en la guía docente de esa asignatura y que ha sido aprobada por la Comisión Académica del Título de Grado de Maestro/a en Educación Infantil. En este caso, se aboga por un modelo docente sociocrítico que promueva sesiones de clase teóricas y prácticas en las que el alumnado cuente con una participación activa y se debata sobre sus representaciones de los principales contenidos de la asignatura. Por ello se va a presentar el cuestionario inicial al que responde la totalidad del alumnado el primer día de clase, como actividad que integra el dossier de actividades que estructura los contenidos especificados por el docente para el tiempo que dura la asignatura (primer cuatrimestre). Después se expondrán los resultados iniciales del cuestionario y se irán discutiendo con los aportes de investigaciones utilizadas en las sesiones de clase, así como con los resultados de otras investigaciones. Finalmente se analizará la concepción final que tiene el alumnado sobre la Didáctica de las Ciencias Sociales al acabar la asignatura.

\subsection{Contexto de la investigación y participantes}

El profesor que suscribe esta investigación ha elaborado y discutido un proyecto docente que amplía y concreta la guía docente de la asignatura Didáctica de las Ciencias Sociales de la Educación Infantil en los tres cursos académicos comprendidos entre septiembre 2017 y enero de 2020. Esta asignatura forma parte de las que componen el bloque didáctico-disciplinar junto con otras que se 
basan en el aprendizaje específico de materias como las Matemáticas, las Ciencias Naturales, las Lenguas (incluyéndose las variantes autonómicas), así como la Música, la Plástica y Educación Física. La materia que nos ocupa tiene una duración de seis créditos ECTS (European Credit Transfer and Accumulation System), lo que la diferencia de las asignaturas del bloque de formación básica (áreas de Psicología, Sociología, Pedagogía...) que tienen una duración de diez créditos ECTS. La incorporación del aprendizaje o didáctica de las Ciencias Sociales a la formación de maestros y maestras ha supuesto la vinculación de los contenidos sociales a la enseñanza (De la Calle, 2008). El resto de asignaturas queda incorporado en esta división en tres bloques (se añade el Practicum y el Trabajo Final de Grado) para el Grado de Maestro/a en Educación Infantil en España, como queda sintetizada en la Tabla 3:

\begin{tabular}{|c|c|}
\hline $\begin{array}{c}\text { Bloque de Formación Básica. Áreas de } \\
\text { Educación, Psicología y Sociología }\end{array}$ & Bloque Didáctico-disciplinar. \\
personalidad & Aprendizaje de las ciencias naturales \\
\hline $\begin{array}{c}\text { Procesos educativos, aprendizaje y desarrollo de la } \\
\text { desarrollo }\end{array}$ & Aprendizaje de las ciencias sociales \\
\hline $\begin{array}{c}\text { Dificultades de aprendizaje y trastornos del } \\
\text { Sociedad, familia y escuela }\end{array}$ & Aprendizaje de las matemáticas \\
\hline Infancia, salud y alimentación & Música, expresión plástica y corporal \\
\hline Organización del espacio escolar, materiales y \\
habilidades docentes
\end{tabular}

Tabla 3. Estructura dual del Plan de Estudios de Maestro/a de Educación Infantil en España. Fuente: Elaboración propia a partir de De la Calle (2008).

Sobre los participantes o alumnado que se encuentra en el cuarto curso del Grado de Maestro/a en Educación Infantil se pueden realizar algunas consideraciones. De los 134 estudiantes a los que se ha encuestado en tres cursos académicos tan solo 10 son varones (Tabla 4), lo que ratifica el predominio de las mujeres por estos estudios universitarios. El $42 \%$ de este alumnado no ha tenido contacto con los contenidos de las Ciencias Sociales (Geografía e Historia) desde la Educación Secundaria o el Bachillerato y el $16 \%$ ha accedido a estos estudios universitarios desde el curso de Técnico de Educación Infantil. Estos datos son relevantes por cuanto interfieren en el recuerdo escolar de las Ciencias Sociales, cuyos contenidos más recientes provienen de asignaturas del bloque de Formación Básica, especialmente aquellas que tienen que ver con la Sociología. 


\begin{tabular}{|c|c|c|c|}
\hline Curso académico & Número de alumnos/as & Varones & Mujeres \\
\hline $2017-18$ & 52 & 3 & 49 \\
\hline $2018-19$ & 38 & 4 & 34 \\
\hline $2019-20$ & 44 & 3 & 41 \\
\hline
\end{tabular}

Tabla 4. Alumnado participante en esta investigación por curso escolar.

El material didáctico se compone de una serie de actividades (ejercicios de reflexión a partir de textos y lecturas) que permiten trabajar los contenidos fundamentales de esa asignatura durante el primer cuatrimestre ( 6 créditos de duración) conjuntamente con el alumnado universitario. La distribución de esos contenidos y su plasmación en actividades para cada uno de los tres bloques temáticos que componen la asignatura queda como sigue en la Tabla 5:

\begin{tabular}{|c|c|c|}
\hline Bloque temático & Principales contenidos & $\begin{array}{c}\mathrm{N}^{\circ} \text { actividades y } \\
\text { sesiones }\end{array}$ \\
\hline $\begin{array}{l}\text { I.-Ciencias Sociales y } \\
\text { Educación Infantil: } \\
\text { fundamentos epistemológicos } \\
\text { y valores educativos }\end{array}$ & $\begin{array}{c}\text { Ciencias Sociales y su didáctica en el } \\
\text { currículo de Educación Infantil. } \\
\text { Medio y entomo. Identidades y ciudadanía. } \\
\text { El hecho social. Pensamiento geográfico y } \\
\text { pensamiento histórico. Educación } \\
\text { emocional y patrimonio. }\end{array}$ & $11 / 17$ \\
\hline $\begin{array}{l}\text { II.-Aspectos metodológicos } \\
\text { en los procesos de enseñanza } \\
\text { y aprendizaje del entomo }\end{array}$ & $\begin{array}{l}\text { Métodos de enseñanza/aprendizaje y } \\
\text { modelos didácticos. } \\
\text { Habilidades sociales para conocer el } \\
\text { entomo. } \\
\text { El juego social y tipologías variadas. } \\
\text { Salidas de campo y paisaje. } \\
\text { Rincones, ambientes y talleres. }\end{array}$ & $6 / 8$ \\
\hline $\begin{array}{l}\text { III.-Programación de los } \\
\text { contenidos: trabajo por } \\
\text { proyectos }\end{array}$ & $\begin{array}{l}\text { Análisis crítico de materiales curriculares. } \\
\text { La educación socioambiental. } \\
\text { Educación para la Paz. } \\
\text { Elaboración de proyectos docentes } \\
\text { globalizados sobre el entomo. }\end{array}$ & $5 / 5$ \\
\hline
\end{tabular}

Tabla 5. Síntesis del proyecto docente con los contenidos, actividades y distribución temporal de sesiones.

Como se aprecia en la tabla anterior el primer bloque de la asignatura dedicado a los contenidos disciplinares es el que más actividades y sesiones de clase ocupa en el proyecto docente. La primera actividad que realiza el alumnado, tras haber sido informado de la metodología de trabajo y el sistema de evaluación, es la realización de la encuesta inicial. En ese momento el alumnado no ha conocido los contenidos de la asignatura, excepto sí por su propia cuenta ha consultado la guía 
docente de la asignatura que está colgada en la web de la facultad. Tras realizar el cuestionario, el docente expone los principales contenidos con la bibliografía correspondiente y una distribución aproximada de las sesiones de clase.

\subsection{El cuestionario como principal instrumento de análisis}

El cuestionario inicial fue respondido por 134 estudiantes universitarios y ha sido validado por un grupo de docentes e investigadores de la Didáctica de las Ciencias Sociales de cinco universidades iberoamericanas. Se compone de once preguntas, de las que las tres primeras responden al modelo test de asociación libre de palabras (Agüero y Chama, 2009; Navia y Estrada, 2012) y las ocho restantes son de respuesta abierta, incluyéndose un dibujo sobre la concepción de la Didáctica de las Ciencias Sociales en la Educación Infantil, como ya se ha hecho en otra investigación similar (Morales y Santana, 2019).

Las preguntas del cuestionario no suelen ofrecer dificultad al alumnado, por lo que suelen responderse en un tiempo medio que ronda los 30 o 40 minutos. Posteriormente se realiza un debate en clase para analizar algunas concepciones, sin que el alumnado pueda modificar sus respuestas. Antes de finalizar esa sesión inicial el profesorado presenta los contenidos de la asignatura, la bibliografía específica y el material docente con el que se desarrollarán las siguientes sesiones de clase hasta finalizar la asignatura.

En la siguiente sesión el docente ya ha analizado las respuestas del cuestionario, especialmente las tres primeras en las que se ha valido del software Evocation 2005. Este software ha sido utilizado en otras investigaciones con representaciones sociales (Lopes, 2010; Silva y Viveiros, 2017) que se han apoyado en las directrices del conocimiento teórico-metodológico sobre este campo de conocimiento (Domingos, 2000; Saraiva, 2007), en consonancia con los estudios del Grupo Midi (Flament, 2001). En esas tres primeras preguntas el alumnado ha escrito cinco palabras que asocia al objeto Didáctica de las Ciencias Sociales en la Educación Infantil (primera pregunta), después las ha numerado de mayor a menor importancia siguiendo una escala numérica de 1 a 5 (segunda pregunta) y en la tercera pregunta ha definido el significado de la palabra a la que ha otorgado mayor valor (valor 1). Estas preguntas junto con el dibujo de lo que entienden por Didáctica de las Ciencias Sociales en la Educación Infantil se vuelven a responder individualmente al finalizar la asignatura para comprobar el grado de conocimiento adquirido durante el período de clases. En el caso de los dibujos se proporciona un espacio acotado para tal fin y el alumnado marca tres emociones de entre diez proporcionadas, que siguen el modelo de Ekman y Friesen (Martínez, 1991). Estos dibujos son semejantes a los esquemas mentales (Kosslyn, 1981) y se han utilizado en la Geografía de la Percepción y del Comportamiento como técnica complementaria a los cuestionarios (Vara, 2010).

Las ocho preguntas restantes ofrecen resultados que se van presentando a lo largo del curso, en relación a los contenidos a tratar en cada una de las actividades (Tabla 6). Estas preguntas se reducen a dos en el cuestionario final (4.- ¿Qué has aprendido en esta asignatura? y 5.- ¿Crees que ha sido importante para tu formación como maestro/a en Educación Infantil? ¿Por qué), puesto que se disponen de otros instrumentos de evaluación (ejercicios de clase, un ejercicio práctico sobre el 


\begin{tabular}{|c|c|}
\hline Aspectos que aparecen en el cuestionario inicial & Preguntas asociadas \\
\hline Recuerdo y utilidad de las Ciencias Sociales & $\begin{array}{l}\text { 5.- ¿Qué son para ti las Ciencias Sociales? ¿Para } \\
\text { qué te han servido? }\end{array}$ \\
\hline $\begin{array}{l}\text { Métodos y recursos utilizados en la enseñanza y } \\
\text { aprendizaje }\end{array}$ & $\begin{array}{c}\text { 6.- ¿Cómo te han enseñado las Ciencias Sociales? } \\
\text { ¿Qué materiales has empleado para estudiar } \\
\text { Ciencias Sociales? }\end{array}$ \\
\hline Preferencia por métodos de enseñanza & $\begin{array}{l}\text { 7.- ¿Cómo te gustaría que te hubiesen enseñado las } \\
\text { Ciencias Sociales? }\end{array}$ \\
\hline Expectativas respecto de la asignatura universitaria & $\begin{array}{l}\text { 8.- ¿Qué esperas de esta asignatura? 9.-¿Crees qué } \\
\text { es importante para tu formación en Educación } \\
\text { Infantil? ¿Por qué? }\end{array}$ \\
\hline $\begin{array}{l}\text { Conocimiento previo de experiencias con las } \\
\text { Ciencias Sociales y propuestas didácticas }\end{array}$ & $\begin{array}{l}\text { 10.- ¿Sabrías conectar algunos de los } \\
\text { conocimientos que has adquirido en el Grado con } \\
\text { esta asignatura? Y, ¿con experiencias de la vida } \\
\text { cotidiana? } \\
\text { 11.- ¿Cuáles son tus propuestas con respecto a la } \\
\text { Didáctica de las Ciencias Sociales de la Educación } \\
\text { Infantil? }\end{array}$ \\
\hline
\end{tabular}

Tabla 6. Preguntas que componen el cuestionario inicial.

\section{Resultados y discusiones: una enseñanza de las Ciencias Sociales mediatizada por su representación social}

Los primeros resultados presentados corresponden a las palabras que el alumnado universitario ha evocado sobre la Didáctica de las Ciencias Sociales en la Educación Infantil, tras haber sido analizados en el software Evocation 2005 con la frecuencia (F) y rango (R) asociados (Tabla 7):

\begin{tabular}{|c|c|c|c|c|c|}
\hline $\mathrm{F} \geq 25 \quad \mathrm{R}<2.5$ & F & $\mathrm{R}$ & $\mathrm{F} \geq 25 \quad \mathrm{R}>2.5$ & $\mathrm{~F}$ & $\mathrm{R}$ \\
\hline Sociedad & 44 & 2,222 & Geografia & 35 & 2,600 \\
\hline Cultura & 37 & 2,000 & Historia & 31 & 2,769 \\
\hline Entorno & 33 & 1,846 & Economía & 26 & 3,625 \\
\hline \multicolumn{3}{|c|}{ (Núcleo central) } & \multicolumn{3}{|c|}{ (Elementos intermedios) } \\
\hline $\mathrm{F} \leq 25 \quad \mathrm{R}<2.5$ & $\mathrm{~F}$ & $\mathrm{R}$ & $\mathrm{F} \leq 25 \mathrm{R}>2.5$ & $\mathrm{~F}$ & $\mathrm{R}$ \\
\hline Familia & 12 & 1,750 & Humanidad & 4 & 3,000 \\
\hline Relaciones & 10 & 1,750 & Normas & 3 & 3,000 \\
\hline Comportamiento & 9 & 2,250 & Relación & 3 & 3,333 \\
\hline Experimentación & 6 & 2,300 & Investigación & 2 & 2,500 \\
\hline Poblaciones & 4 & 1,667 & Arte & 2 & 2,500 \\
\hline Valores & 4 & 2,333 & & & \\
\hline \multicolumn{3}{|c|}{ (Elementos periféricos) } & \multicolumn{3}{|c|}{ (Elementos periféricos) } \\
\hline
\end{tabular}

Tabla 7. Estructura representacional del alumnado sobre la didáctica de las Ciencias Sociales en la Educación Infantil al inicio de la asignatura. 
Los datos de la Tabla 7 revelan que la Didáctica de las Ciencias Sociales en la etapa de Educación Infantil se asocia al estudio de la sociedad, la cultura y el entorno. Esas tres palabras forman parte del núcleo central de la representación del alumnado universitario por haber sido evocadas con mayor frecuencia y rango. Se trata de términos genéricos pero que tienen una relación entre sí (especialmente la sociedad y el entorno), como demuestran algunas definiciones dadas por el alumnado en las que aflora la socialización: "pienso que la palabra sociedad engloba las ciencias sociales" (P029M); "considero que en las ciencias sociales uno de los aspectos que más se trabaja es la sociedad, ya que pueda englobar todo aquello que nos rodea" (P048M) y "porque las ciencias sociales engloban todo aquello que significa socializarse con las personas, comprender que todos somos diferentes, pero al mismo tiempo iguales" (P081M). Cuando el alumnado se ha referido a la cultura ha establecido relaciones con las Ciencias Sociales (Geografía e Historia) que han aparecido como elementos intermedios: "no sé lo que voy a estudiar en esta asignatura, pero las culturas engloban tanto la situación geográfica como las diferencias entre géneros, diferentes países, etc." (P0104M). La sociedad concentra el valor simbólico y el poder asociativo (en términos de Sá, 2002) y es el contenido que mejor define la asignatura, en la que se distingue entre un contenido descriptivo (la familia, las relaciones sociales, las poblaciones) y un contenido valorativo (el comportamiento y los valores), que aparecen como elementos intermedios asociados a la estructura del núcleo duro.

Del análisis de los dibujos de la cuarta pregunta del cuestionario se desprende una concepción mayoritariamente social de la disciplina $(59,3 \%)$, con escasas interdependencias con las esferas física y natural $(22,6 \%)$, que conocen por otras asignaturas del plan de estudios. Eso repercute en las propuestas didácticas que han formulado con un escaso carácter inter o multidisciplinar $(21,7 \%)$, coincidiendo con los resultados homónimos (25,2\%) de Morales y Santana (2019), y es coherente con las asignaturas que más han nombrado en el cuestionario (Estructura social y educación; Sociología de la Educación) y que relacionan con la Didáctica de las Ciencias Sociales. Con estos mismos autores se equiparan los datos sobre la falta de representación de metodologías docentes en los dibujos (en un $44,5 \%$ de ese estudio no lo han representado y un $39,4 \%$ en esta investigación), por lo que unido a la fuerte presencia de la figura docente en actitud de transmitir conocimientos se induce la reproducción de un modelo tradicional o técnico, tal y como han señalado otras investigaciones (Apple, 2000; Hernández, 2013, 2014; Trueba, 1997). Las mínimas referencias a modelos alternativos o de investigación tienen una estrecha relación con la posición del concepto investigación en el cuadrante de los elementos periféricos de la Tabla 7.

El recuerdo escolar de las Ciencias Sociales se asocia a los materiales de Geografía e Historia de la Educación Secundaria y del Bachillerato, así como ocasionalmente al conocimiento del medio de la Educación Primaria. La enseñanza recibida en esas materias se rememora mediante referencias a la memorización, la escasa utilidad posterior y el uso de recursos (Figura 1). Casi el $60 \%$ del alumnado ha mencionado los mapas como el principal recurso de sus clases de Ciencias Sociales, lo que unido al uso del libro de texto representa más del $70 \%$ del recuerdo sobre los materiales didácticos. Estos datos están en sintonía con lo que se ha señalado en otras investigaciones que han analizado el recuerdo escolar de esas materias (Fernández-Caso et al., 2010; Fuentes, 2004; Martínez et al., 2006; Pagès, 1996; Parra, 2019; Souto y García-Monteagudo, 2019). 


\section{Recursos utilizados en la enseñanza de las Ciencias Sociales}

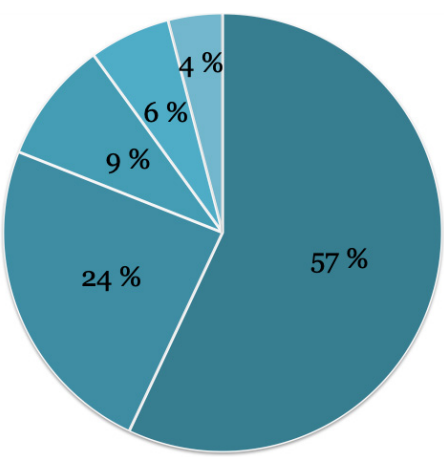

Mapas

Libros de texto

Apuntes del profesor

Salidas de campo

No sabe / No contesta

Figura 1. Recuerdo escolar sobre los recursos didácticos utilizados en las clases de Ciencias Sociales.

La consecuencia de ese recuerdo negativo de las Ciencias Sociales es que la expectativa de la asignatura universitaria y los métodos de enseñanza sean más activos y adquieran un carácter más práctico y globalizado, que esté orientado a la actuación concreta en el aula de Educación Infantil (Tabla 8). En ningún caso se han mencionado métodos de investigación ni el conocimiento de propuestas innovadoras en el área de Ciencias Sociales.

\begin{tabular}{|c|c|}
\hline Expectativas de la asignatura universitaria (\%) & Unidades de significado \\
\hline $\begin{array}{c}\text { Que tenga un carácter práctico y fomente la } \\
\text { participación (54.2\%) }\end{array}$ & $\begin{array}{c}\text { "Aprender ciencias sociales de forma práctica, } \\
\text { siendo nosotros los que construyamos nuestro } \\
\text { propio aprendizaje mediante la lectura y reflexión } \\
\text { en el aula" (P061M). }\end{array}$ \\
\hline $\begin{array}{c}\text { Que aporte recursos de aplicación al aula de } \\
\text { Educación Infantil (28.7\%) }\end{array}$ & $\begin{array}{c}\text { "Recursos que me faciliten la enseñanza con los } \\
\text { más pequeños" (P027M). }\end{array}$ \\
\hline Que sea lúdica (16.3\%) & $\begin{array}{c}\text { "Aprender nuevos conocimientos relacionados con } \\
\text { la socialización y saber enseñarlos de forma lúdica } \\
\text { y globalizada" (P093M). }\end{array}$ \\
\hline No sabe/No contesta (0.8\%) & \\
\hline
\end{tabular}

Tabla 8. Expectativas del alumnado al iniciar la asignatura universitaria.

A lo anterior se añaden las propuestas iniciales que el alumnado considera que deben guiar las clases universitarias en dicha asignatura. No suelen ser propuestas didácticas que hayan formulado de manera concreta, salvo un $12 \%$ del futuro profesorado que ha mencionado algunas temáticas de tipo transversal (el reciclaje, seguridad vial, medios de comunicación...). Más bien son deseos de conseguir recursos prácticos para "saber aplicar los conocimientos de las Ciencias Sociales en las aulas de Educación Infantil y, además, hacer que esta área sea un aprendizaje global, de experimentación y de interés para los niños y niñas de estas edades" (P037M). Las palabras de esta alumna coinciden genéricamente con lo apuntado por Barton, McCully y Marks (2004) acerca de las preocupaciones del profesorado en formación. 
Tras el desarrollo de las sesiones docentes de cada uno de los bloques temáticos que componen la asignatura, se fueron realizando diferentes actividades, ejercicios prácticos y se desarrollaron las propuestas didácticas. El último día del curso el alumnado respondió el cuestionario y los resultados de la estructura representacional son los que siguen a continuación (Tabla 9):

\begin{tabular}{|c|c|c|c|c|c|}
\hline $\mathrm{F} \geq 31 \quad \mathrm{R}<2.5$ & $\mathrm{~F}$ & $\mathrm{R}$ & $\mathrm{F} \geq 31 \mathrm{R}>2.5$ & $\mathrm{~F}$ & $\mathrm{R}$ \\
\hline Ciudadanía & 39 & 2,462 & Medio & 35 & 3,2 \\
\hline Tiempo & 36 & 2,273 & Entorno & 33 & 2,667 \\
\hline Espacio & 34 & 2,220 & & & \\
\hline Identidad & 32 & 1,625 & & & \\
\hline \multicolumn{3}{|c|}{ (Núcleo central) } & \multicolumn{3}{|c|}{ (Elementos intermedios) } \\
\hline $\mathrm{F} \leq 31 \quad \mathrm{R}<2.5$ & $\mathrm{~F}$ & $\mathrm{R}$ & $\mathrm{F} \leq 31 \quad \mathrm{R}>2.5$ & $\mathrm{~F}$ & $\mathrm{R}$ \\
\hline Socialización & 18 & 2,000 & Cultura & 9 & 3,667 \\
\hline Sociedad & 15 & 2,250 & Práctica & 7 & 3,333 \\
\hline Cooperación & 13 & 1,667 & Inclusión & 6 & 3,000 \\
\hline Aprendizaje & 13 & 2,000 & & & \\
\hline Valores & 9 & 2,330 & & & \\
\hline \multicolumn{3}{|c|}{ (Elementos periféricos) } & \multicolumn{3}{|c|}{ (Elementos periféricos) } \\
\hline
\end{tabular}

Tabla 9. Estructura representacional del alumnado sobre la didáctica de las ciencias sociales en la Educación Infantil al final de la asignatura.

Los datos de la Tabla 9 indican que la estrategia docente ha tenido resultados en el aprendizaje de las Ciencias Sociales y su didáctica en la etapa de Infantil. Las palabras del núcleo central constituyen los principales contenidos teóricos (la ciudadanía, espacio geográfico, tiempo histórico e identidades) debatidos en el primer bloque de la asignatura a partir de las definiciones de autores de referencia (Aranda, 2016; Benejam 1993; Hernández, 2008; Souto, 1999) y que han tenido su aplicación metodológica y didáctica en el resto de la asignatura. Los contenidos sobre medio y entorno son indicadores de la importancia del contexto y marcan la transición hacia las finalidades de las Ciencias Sociales (socialización, valores, cultura...), sin renunciar al aprendizaje y la cooperación. Con todo ello el alumnado ha mostrado evidencias más claras de la importancia de esta asignatura en su formación docente, destacándose especialmente la utilidad que tendrán los contenidos en su posterior desempeño como maestros y maestras de Educación Infantil. La siguiente respuesta de una alumna es un buen indicador de este cambio de representación: "Sí, ha sido muy importante. No solo por la materia entendida como contenido teórico en sí, sino como modo de entender la educación infantil, de saber buscar información y modos diferentes de trabajar, y cómo ponerlos en práctica" (P0123M).

Todos estos elementos han estado presentes en las veintisiete propuestas didácticas grupales que el alumnado ha elaborado para esta asignatura: las temáticas de elección libre al inicio de la asignatura han abordado aspectos como el agua, la alimentación, la cultura egipcia, el medio local y la familia. Como temáticas más innovadoras se pueden citar "Llamando al pasado" sobre los medios de comunicación, "Luces y sombras" acerca de la influencia de los avances tecnológicos en la sociedad actual y "1,2,3...iAcción!" sobre el cine. Tampoco han faltado las referencias a la identidad de género ("Pretty Woman") y al arte ("Un viaje por el arte"), dos aspectos que no se mencionaban en la representación inicial del alumnado. Sobre estas propuestas didácticas se dispone de rúbricas 
de evaluación y se han ido elaborando durante los cursos académicos correspondientes, junto con otras actividades en las que se ha ido experimentado un cambio relevante con el que concluiremos en el siguiente apartado.

\section{Conclusiones}

La utilización de la teoría de las representaciones sociales como marco de investigación para la formación docente ha permitido aproximarnos a los cambios de concepciones sobre la didáctica de las ciencias sociales. Los resultados deben interpretarse en el contexto de un estudio de casos, pero la abundancia de técnicas y recursos analizados permite que se puedan extrapolar a otras situaciones similares. En el transcurso de la asignatura y como ha evidenciado el cuestionario, el profesorado en formación ha logrado cambiar los aspectos más negativos de su representación inicial de las Ciencias Sociales hacia una concepción más dinámica y participativa en la que han concedido mayor importancia tanto a la asignatura del grado universitario, como al área de conocimiento. El cambio en las palabras del núcleo central de la representación de la asignatura entre el primer y el último cuestionario refleja que el alumnado ha asumido nuevos conceptos de las Ciencias Sociales que ha terminado usando como propios. Es el caso de las identidades y la ciudadanía, que al iniciar los cursos académicos se percibían como abstractos y no se relacionaban con esta asignatura. Al finalizar la asignatura, saben explicar la importancia de estos conceptos y su trascendencia en la educación del alumnado de Educación Infantil. Con respecto a los contenidos propios del pensamiento geográfico e histórico, han comprobado que la Geografía y la Historia pueden aprenderse desde edades tempranas, un aspecto que ha minimizado notoriamente su visión negativa de estas disciplinas respecto a su recuerdo escolar.

Las expectativas del alumnado universitario se han cumplido y han sido capaces de realizar propuestas didácticas sobre temas relacionados con las Ciencias Sociales, sus contenidos específicos y sus finalidades (acceso a una ciudadanía intercultural, socialización y el desarrollo de la personalidad en un contexto global). Además, lo han hecho desde una posición socioconstructivista y se ha ido asumiendo una visión más crítica con los contenidos curriculares que les ha causado cierto descontento a medida que han ido descubriendo las posibilidades didácticas de estas ciencias en el segundo ciclo de Educación Infantil. Por tanto, se puede afirmar que la mayoría de este futuro profesorado finaliza esta asignatura con menor rechazo a la implementación didáctica de los contenidos en Educación Infantil, pues es capaz de defender la utilidad de estas ciencias, incluso relacionarlas con otros campos de conocimiento (especialmente las ciencias naturales, la música y algunas lenguas) para elaborar proyectos didácticos interdisciplinares.

A medio plazo el alumnado consigue adquirir una serie de competencias que constan en la guía docente de la asignatura y desarrolla su capacidad crítica para debatir e iniciarse en la investigación que les será necesaria en la elaboración del Trabajo Final de Grado, como muestra la siguiente alumna: "Finalmente, pienso que esta asignatura me ha hecho abrir la mente como docente y como persona. Gracias a ella me he parado a reflexionar sobre ideas en las que antes ni pensaba. Además, a parte de los conocimientos obtenidos sobre las Ciencias Sociales, he aprendido otros aspectos también muy necesarios como: mejorar mi redacción, aprender a buscar información, y, la asignatura pendiente de la mayoría, citar y referenciar" (P014M). Es, por tanto, una asignatura útil e importante en su formación docente, que una parte del alumnado reivindica que aparezca antes (en los primeros cursos del plan de estudios) en su formación universitaria. 
En cualquier caso, es conveniente seguir midiendo los resultados adquiridos a largo plazo. Esto supone que se deben realizar entrevistas semiestructuradas y/o grupos de discusión con el alumnado cuando ya es graduado/a en magisterio, pues de esa manera se comprobará si sus concepciones se han consolidado y podrá ejercer una docencia más crítica y ajustada a la formación ciudadana de su futuro alumnado. Así es como continuaremos investigando en los próximos cursos académicos para mejorar la innovación docente en el ámbito de la Didáctica de las Ciencias Sociales.

\section{Referencias}

Agüero, A. y Chama, M. (2009). Arriesgando la palabra: cultura y psicoanálisis. Autores de Argentina. Apple, M. W. (2000). Official knowledge (2. ${ }^{a}$ edición). Routledge.

Aranda, A. Ma . (2016). Didáctica de las Ciencias Sociales en la Educación Infantil. Síntesis.

Barton, K. C. y Levstik, L. S. (2004). Teaching history for the common good. Lawrence Erlbaum.

Barton, K. C., McCully, A. W. y Marks, M. J. (2004). Reflecting on elementary children's understanding of history and social studies: An inquiry project with beginning Teachers in Northern Ireland and the United States. Journal of Teacher Education, 55(1), 70-90.

https://doi.org/10.1177/0022487103260069

Benejam, P. (1993). La didàctica de la geografia des de la perspectiva constructivista. Documents d'Anàlisi Geogràfica, 21, 35-52. https://raco.cat/index.php/DocumentsAnalisi/article/view/41557 Bugental, D. B. y Goodnow, J. J. (1998). Socialization processes. En W. Damon y N. Eisenberg (Eds.), Handbook of child psychology: Social, emotional and personality development (pp. 389462). John Wiley \& Sons Inc.

Calvani, A. (1988). Il bambino, il tempo, la storia. La Nuova Italia.

Canella, G. S. y Radhika, V. (2005). Childhood and postcolonization. RoutledgeFalmer.

Canet, S., Morales, A.J. y García-Monteagudo, D. (2018). Pensar geográficamente en la Educación Infantil: de la imaginación a la construcción social del espacio concebido. Didáctica Geográfica, 19, 23-46. https://dialnet.unirioja.es/servlet/articulo? codigo $=7389434$ \&orden $=0 \& i n f o=l i n k$

Capel, H. (1973). Percepción del medio y comportamiento geográfico. Revista de Geografía, 7, 58-150. https://dialnet.unirioja.es/servlet/articulo? codigo $=2856533$

Casiello, M. (2012, 20 de septiembre). ¿Por qué enseñar ciencias sociales en Educación Infantil? Lic. María de los Ángeles CASIELLO. https://bit.ly/37teqBL

Castorina, J. A. (2017). Las representaciones sociales y los procesos de enseñanza-aprendizaje de conocimientos sociales. Psicología da Educação, 44, 1-13. https://doi.org/gqmz

Catling, S. (1993). The whole world in our hands. Geography, 78(4), 340-358. https://www.jstor.org/stable/40572549

Chartier, R. (1996). El mundo como representación. Historia cultural. Entre la práctica y la representación. Gedisa.

Cooper, H. (2002). Didáctica de la historia en la educación infantil y primaria. Morata.

Corsaro, W. (1997). The sociology of childhood. Pine Forge Press.

Cuenca, J. (2008). La enseñanza y el aprendizaje de las ciencias sociales en educación infantil en R. Ma Ávila, A. Cruz y Mª . C. Díez (Coords.), Didáctica de las Ciencias Sociales, currículo escolar y formación del profesorado: la didáctica de las Ciencias Sociales en los nuevos planes de estudios (pp. 289-311). Universidad de Jaén y Asociación Universitaria del Profesorado de Didáctica de las Ciencias Sociales. 
De la Calle, M. (2008). La formación del profesorado de Educación Infantil y la didáctica de las ciencias sociales en el EEES. En R. Mª . Ávila, A. Cruz y Mª . C. Díez (Eds.), Didáctica de las Ciencias Sociales, currículo escolar y formación del profesorado: La didáctica de las Ciencias Sociales en los nuevos planes de estudios (pp. 63-78). Universidad de Jaén y Asociación Universitaria del Profesorado de Didáctica de las Ciencias Sociales.

Domingos, M. (2000). Habitus e representações sociais: questões para o estudo de identidades coletivas. En A. S. P., Moreira y D. C. Oliveira (Orgs.), Estudos interdisciplinares de representação social (pp. 117-159). AB.

Donet, E. (2016). Pensar històricament en Educació Infantil. Un estudi de cas (Trabajo Final de Máster no publicado).Universitat de València, València.

Feliú, M. y Jiménez, L. (2015). Ciencias sociales y educación infantil (3-6). Cuando despertó el mundo estaba allí. Graó.

Fernández-Caso, $M^{a}$. V., Guverich, R., Souto, P., Bachmann, L., Ajón, A. y Quintero, S. (2010). La imagen pública de la geografía. Una indagación desde las visiones de profesores y padres de alumnos secundarios. Biblio 3W, Revista Bibliográfica de Geografía y Ciencias Sociales, vol. XV, 859. http://www.ub.edu/geocrit/b3w-859.htm

Flament, C. (2001). Estrutura e dinâmica das representações sociais. En D. Jodelet (Org.), As representações sociais. Traducción Lílian Ulup (pp. 173-186). EdUERJ.

Fuentes, C. (2004). Concepciones de los alumnos sobre la Historia. Enseñanza de las Ciencias Sociales, 3, 75-83. https://dialnet.unirioja.es/servlet/articulo?codigo $=820733$

Giroux, H. A. (1998). Public pedagogy and rodent politics: Cultural studies and the challenge of Disney. Arizona Journal of Hispanic Cultural Studies, 2, 253-266. shorturl.at/azPR2

Gómez, A. E. y Núñez, P. (2006). Formar para investigar, investigar para formar en Didáctica de las Ciencias Sociales. dialnet.unirioja.es/servlet/libro?codigo $=500427$

Hernández, F.X. (2008). Didáctica de las ciencias sociales, geografía e historia. Graó.

Hernández, L. (2013). La enseñanza de las ciencias sociales en la formación profesional de las estudiantes de maestra de educación infantil (Tesis doctoral), Universitat Autònoma de Barcelona. http://hdl.handle.net/10803/125964

Hernández, L. (2014). ¿Quiénes enseñan el medio social en la Educación Infantil? Representaciones sociales sobre los modelos de profesora de Educación Infantil para la enseñanza de las ciencias sociales. Revista Latinoamericana de Estudios Educativos, 1(10), 113-135.

https://www.redalyc.org/pdf/1341/134139791006.pdf

Hernández, L. y Pagès, J. (2014). La enseñanza de las ciencias sociales en la Educación Infantil en México: las representaciones sociales y la práctica docente de nueve estudiantes de maestra de educación infantil. Educação em Revista, 3o(1), 65-94. https://doi.org/gfomcb

James, A., Jenks, C. y Prout, A. (1998). Theorizing childhood. Polity Press.

Jodelet, D. (2009). Recentes desenvolvimentos da noção de representações nas ciências sociais. En Â. M. Almeida y Denise Jodelet (Orgs.), Interdisciplinaridade e diversidade de paradigmas Representações sociais (pp. 105-122). Thesaurus.

Kosslyn, S. (1981). El medio y el mensaje en las imágenes mentales: una teoría. Revisión Psicológica, 88(1),46-66. Lobato, A. (2013). Representaciones sociales y didáctica: construcción teórica de un espacio común. Magis, Revista Internacional de Investigación en Educación, 5(11), 277-295. https://doi.org/10.11144/Javeriana.m5-11.rsdc

Lopes, M.E. (2010). Praxiologia, representação social de menopausa e práticas educativas de enfermeiras na estratégia saúde da família. (Tesis doctoral no publicada). Universidade Federal do Río Grande do Norte, Río Grande do Norte. 
Maestro, P. (2002). El modelo de las historias generales y la enseñanza de la historia. Didáctica de las Ciencias Experimentales y Sociales, 16, 3-33. https://bit.ly/2Vw4ips

Martin, F. y Owens, P. (2005). Young children making sense of the world. En S. Scoffham (Ed.), Primary geography handbook (pp. 63-73). Geographical Association.

Martínez, J. (1991). Las emociones y su expresión en la primera infancia. Las vías facial y vocal. Revista de Pedagogía de la Universidad de Salamanca, 4, 65-82.

https://revistas.usal.es/index.php/0214-3402/article/view/3250

Martínez, N., Souto, X. M. y Beltrán, J. (2006). Los profesores de historia y la enseñanza de la historia en España. Una investigación a partir de los recuerdos de los alumnos. Enseñanza de las Ciencias Sociales: Revista de Investigación, 5, 55-71. https://dialnet.unirioja.es/servlet/articulo?codigo=1700714

Massey, D. (2005). For space. Sage Publications.

Mateos, J. (2001). Genealogía del código pedagógico del entorno. Aula, 13, 19-35. https://doi.org/10.14201/3590 Mateos, J. (2008). Globalización del conocimiento escolar: genealogía y problemas actuales. Didáctica de las Ciencias Experimentalesy Sociales, 22, 3-22. https://ojs.uv.es/index.php/dces/article/view/2421

Morales, A.J. y Santana, D. (2019). Las representaciones de la didáctica de las ciencias sociales en los futuros docentes de educación infantil. En D. Parra y C. Fuertes (Coords.), Reinterpretar la tradición, transformar las prácticas: Ciencias sociales para una educación crítica (pp. 99-121). Tirant lo Blanch.

Moscovici, S. (2007). Representações sociais. Investigação em psicologia social. Vozes.

Moscovici, S. y Hewstone, M. (1986). De la ciencia al sentido común. En S. Moscovici (Eds.), Psicología Social II. Pensamiento y vida social. Psicología social y problemas sociales (pp. 679-710). Paidós.

Navia, M. y Estrada, H. (2012). Uso de la técnica de asociación libre para conocer la percepción del consumidor sobre queso costeño en Colombia. Psicogente, 15(28), 271-286.

http://revistas.unisimon.edu.co/index.php/psicogente/article/view/1875

Ortega, P. (1996). Valores y educación. Ariel.

Pagès, J. (1994). La didáctica de las ciencias sociales, el currículo y la formación del profesorado. Signos, Teoría y Práctica de la Educación, 5(13), 38-51. https://dialnet.unirioja.es/descarga/libro/2910oo.pdf

Pagès, J. (1996). Las representaciones de los estudiantes de maestro sobre la enseñanza de las Ciencias Sociales: ¿̇cuáles son?, ¿̇cómo aprovecharlas? Investigación en la escuela, 28, 103-114.

https://doi.org/10.12795/IE.1996.i28.08

Palmer, J. y Birch, J. (2004). Geography in the early years. Routledge.

Parra, D. y Segarra, J.R. (2011). Cultura y pertenencia: el tratamiento didáctico de contenidos histórico-culturales en las aulas valencianas de Educación Infantil y Primaria. Didáctica de las Ciencias Experimentales y Sociales, 25, 65-83. https://bit.ly/3rTsAWt

Parra, D. (2019). Representación de la historia escolar y crítica del consenso de sentido común. En D. Parra y C. Fuertes (Coords.), Reinterpretar la tradición, transformar las prácticas. Ciencias Sociales para una educación crítica (pp. 99-121). Tirant lo Blanch.

Pillet, F. (2004). La geografía y las distintas acepciones del espacio geográfico. Investigaciones Geográficas, 34, 141-154. investigacionesgeograficas.com/issue/view/2004-n34

Pontes, A., Serrano, R. y Poyato, F. J. (2012). Concepciones y motivaciones sobre el desarrollo profesional docente en la formación inicial del profesorado de educación secundaria. Eureka. Revista sobre Enseñanza y Divulgación de las Ciencias, 1O, 533-551. http://hdl.handle.net/10498/15612

Porlán R., Rivero, A. y Martín, R. (1998). Conocimiento profesional y epistemología de los profesores I: Teoría, métodos e instrumentos. Enseñanza de las Ciencias: Revista de Investigación y Experiencias Didácticas, 15(2), 155-71. https://raco.cat/index.php/Ensenanza/article/view/21488 
Raths, H. (1976). El sentido de los valores y la enseñanza. Cómo emplear los valores en el Salón de Clase. Unión Tipográfica Editorial.

Real Decreto 1630/2006, de 29 de diciembre, por el que se establecen las enseñanzas mínimas del segundo ciclo de Educación Infantil. https://www.boe.es/eli/es/rd/2006/12/29/1630

Rivero, M. P. (2011). Didáctica de las Ciencias Sociales para la Educación Infantil. Mira Editores.

Rivero, M. P. y Gil. A. (2011). Pensar y expresar el espacio en el aula de Infantil. En Ma . P. Rivero (Coord.), Didáctica de las Ciencias Sociales para Educación Infantil (pp. 31-47). Mira Editores.

Rodríguez, F. (1995). Didáctica das ciencias sociais na educación infantil. Indo Edicións.

Sá, C. P. (2002). Núcleo central das representações sociais. Vozes.

Sammut, G., Andreouli, E., Gaskell, G. y Valsiner, J. (2015). Social representations: a revolutionary paradigm? En G. Sammut, E. Andreouli, G. Gaskell y J. Valsiner (Eds.), Cambridge handbook of social representations (pp. 3-11). Cambridge University Press.

Santisteban, A. (2005). Les representacions i l'ensenyament del temps històric. Estudis de cas en formació inicial de mestres de primària en Didàctica de les Ciències Socials (Tesis doctoral), Universitat Autònoma de Barcelona. Recuperado de Tesis Doctorals en Xarxa http://hdl.handle.net/10803/4666

Saraiva, J. (2007). Habitus docente e representaçao social do "ensinar geografia” na Educaçao Básica de Teresina-Piauí. Programa Posgraduaçao em Educaçao. Universidade Federal Río Grande do Norte.

Sebastià, R. (2014). Ideas previas y aprendizaje significativo en la enseñanza de la geografía (pp. 1574). En R. Martínez y E. Ma ${ }^{\mathrm{a}}$. Tonda (Eds.), Nuevas perspectivas conceptuales y metodológicas para la educación geográfica. Volumen I. Área de Didáctica de las Ciencias Sociales de la Universidad de Córdoba. Grupo de Didáctica de la Geografía de la Asociación de Geógrafos Españoles. http://rua.ua.es/dspace/handle/10045/49187

Silva, E. A. da y Viveiros, K. F. M. (2017). Representações sociais de pobreza construídas pelos cursistas da Especialização Educação, Pobreza e Desigualdade Social realizada no Rio Grande do Norte: primeiros resultados. Educar em Revista, 33(2), 35-54. https://doi.org/10.1590/0104-4060.51389

Sinkin, H.y Becerra, G. (2013). El proceso de socialización: apuntes para su exploración en el campo psicosocial. Ciencia, Docencia y Tecnología, 24(47), 119-142. dialnet.unirioja.es/servlet/articulo?codigo=4696738

Souto, X. M. (1999). Didáctica de la geografía. Problemas sociales y conocimiento del medio. Ediciones del Serbal.

Souto, X. M. y García-Monteagudo, D. (2019). Conocer las rutinas para innovar en la geografía escolar. Revista de Geografía Norte Grande, 74, 207-228. https://doi.org/gx75

Tonda, E. M. (2001). La didáctica de las ciencias sociales en la formación del profesorado de educación infantil. Universidad de Alicante.

Tonda, E. M. (2009). La geografía en el grado de maestro de educación infantil en España. En VV.AA. (Coord.), A Inteligência Geográfica na Educação do Século XXI (pp. 1-23). Associação de Professores de Geografia.

Trepat, C. A. (2011). El aprendizaje del tiempo en educación infantil. En M. P. Rivero (Coord.), Didáctica de las Ciencias Sociales para Educación Infantil (pp. 49-63). Mira Editores.

Trueba, B. (1997). Modelos didácticos y materiales curriculares en Educación Infantil. Investigación en la Escuela, 33, 35-46. https://revistascientificas.us.es/index.php/IE/article/view/7931/7023

Vara, J. L. (2010). Un análisis necesario: epistemología de la geografía de la percepción. Papeles de Geografia, 51-52, 337-344. https://revistas.um.es/geografia/article/view/114631

Wood, L. y Holden, C. (2007). Ensenyar història al més petits. Zenobita Edicions. 